\title{
PEER EDUCATION INTERVENTION ON ADOLESCENTS' ANXIETY, DEPRESSION, AND SLEEP DISORDER DURING THE COVID-19 PANDEMIC
}

\author{
Xiushi Ding \& Jiwei Yao \\ School of Sports and Health, Guangxi Normal University, Guilin, Guangxi, China
}

received: 14.5 .2020

revised: 7.9 .2020

accepted: 29.9.2020

\begin{abstract}
SUMMARY
Background: Adolescents' anxiety and depression during the coronavirus disease 2019 (COVID-19) pandemic outbreak cannot be ignored. In public health crisis events, adolescents are prone to negative psychological problems, such as anxiety and depression. Hence, this research focuses on the use of reasonable and efficient methods to intervene in adolescents' psychological problems during the COVID-19 pandemic.

Subjects and methods: From February to April 2020, we conducted an anonymous online survey on a total of 1,200 adolescents in the provinces of Hunan and Guangxi in China. Moreover, we randomly divided a total of 150 middle school students with anxiety scores greater than 50 and volunteered to participate in the intervention experiment into control and intervention groups, with 75 members in each group. On the basis of the proposed routine treatment, we conducted 8 weeks of model 328-based peer education intervention in the intervention group.

Results: After the intervention, the self-rating anxiety scale scores (SAS) of the intervention group are better than those of the control group $(P<0.001)$. Moreover, the self-rating depression scale $(S D S)$ scores of both groups are reduced, but the effect is more significant on the intervention group $(P<0.001)$ than on the control group. Finally, the total Pittsburgh sleep quality index (PSQI) scores of both groups are reduced, but the effect is more significant on the intervention group than on the control group ( $P=0.001$ and $<0.001$, respectively).

Conclusions: Model 328-based peer education intervention can significantly reduce the level of anxiety and depression in adolescents and improve their sleep quality.
\end{abstract}

Key words: model 328 - peer education - COVID-19 pandemic - anxiety - sleep disorder - depression

$* * * * *$

\section{INTRODUCTION}

Coronavirus disease 2019 (COVID-19) is currently a major public health event of international concern. Given the absence of vaccine against COVID-19 and its nature of extensive dissemination and transmission, it will be life-threatening to affected individuals who do not receive timely treatment (Joseph et al. 2020). With the emergence of media information, adolescents are likely to suffer from a huge psychological attack and influence. Furthermore, media information is flooded, causing a huge effect on the public's psychology. Jakovljevic et al. (2020) found that the prevalence of anxiety, depression, and sleep disorder is significantly higher among younger groups than older groups. For the susceptible group of adolescents, their school curriculum and daily activities have been conducted indoors since the COVID-19 outbreak (Juan et al. 2020). This lifestyle change and the potential risk of infection, together with the imperfect development of adolescents' own emotional regulation and control capabilities, may lead to serious anxiety and depression problems among adolescents.

The mental health of adolescents has been extensively investigated, especially by scientific researchers (Danese et al. 2020). Adolescents' psychological problems dominated by anxiety and depression have become indicators of their mental health conditions
(Tuliao et al. 2020). Compared with other psychological problems, anxiety disorder has a relatively early onset and an extremely high prevalence among adolescents. Anxiety and depression in adolescents are susceptible to the influence of external social environments, including sudden public crisis events (Holmes et al. 2020). Ghandour et al. (2019) found that the prevalence of anxiety among adolescents is $7.1 \%$ in US. A relevant epidemiological survey in China shows that the prevalence of anxiety is $3.5 \%$ among middle school students in Changsha, Hunan Province, China (Guan et al. 2010). Anxiety has a negative effect on adolescents' self-development and social life. Studies show that social anxiety is one of the most common anxieties among adolescent groups. Adolescents who have been suffering from social anxiety for a long time have the characteristics of low self-esteem and low social self-efficacy, which are not conducive to the development of positive self-concept (Bowles 2017). Anxiety is also an antecedent variable that reduces adolescents' academic self-efficacy and negatively affects their academic achievements (Pekrun 2017). Follow-up studies also show that anxiety can also predict Internet addiction in adolescents one year later, and those with excessive anxiety are likely to suffer Internet addiction after one year (Stavropoulos et al. 2017). In addition to anxiety, the prevalence of depression is also high among teenagers (Stein et al. 
2018). Studies confirm that adolescents with severe depression face a high incidence of substance abuse (Mason et al. 2019), poor academic performance (Davis et al. 2018), and family function disorder, which in turn increase their depression and even lead to suicide (Yeh et al. 2016). Sleep disorder is the core symptom for anxiety and depression. Follow-up studies indicate that it also predicts the level of anxiety and depression in the future, suggesting that there may be an interactive relationship between emotional disorder and sleep disorder (Shanahan et al. 2014).

Relevant studies confirm that exercise intervention has a positive effect on physical and mental health. Brosnahan et al. (2004) explored the relationship between physical exercise and mental health among adolescents. They pointed out that exercise can reduce adolescents' pessimism and suicidal behavior, and it can be used as an intervention to improve adolescents' mental health. Tull et al. (2018) revealed that physical exercise can reduce anxiety and depression and has a certain therapeutic effect on both mental health disorders. Lubans et al. (2010) conducted an exercise intervention on students to analyze their degree of anxiety. The results showed that certain exercise intervention can effectively improve students' awareness of independent exercise and reduce adolescents' anxiety levels. The experiment by Zhao et al. (2016) proved that exercise intervention can effectively eliminate the extent and depth of psychological depression and has an intervention effect on each factor, especially on sleep disorder, anxiety/somatization, and retardation factors. Relevant researchers conducted considerable empirical studies on the relationship between exercise intervention and mental health (Ramirez et al. 2018). Their results showed that planned and purposeful exercise interventions can regulate individuals' psychological activities and promote their mental health. However, according to existing research on exercise intervention, restricted exercise venues and insufficient exercise episodes are undesirable factors influencing the intervention effect. On this basis, we adopt model 328 as the exercise intervention model in this study. Model 328 is a brand-new exercise model proposed and supported by the Health Qigong Management Center of the General Administration of Sports of China in accordance with the "National Physical Training Standards" (Xu 2019). The specific implementation method is described as follows: individuals improve their health by exercising three days a week, twice a day, and eight movements each time. The exercise programs in this model include Tai Chi, free-hand fitness, gymnastic qigong, yoga, and other simple exercises. Compared with other exercise models, model 328 is designed on the basis of people's lifestyles and exercise habits. At present, studies confirm that the advantages of model 328 include non-restricted exercise time and venues, including parks, offices, classrooms, and other places. Model 328 can improve people's physical and mental health and help them develop a good lifestyle. To the best of our knowledge, only a few studies combine model 328 with psychological intervention.

Peer education is a model of health education that refers to the education form in which individuals share information, ideas, or behavioral skills because they have certain similarities for different reasons, such as age, gender, living environment and experience, and cultural and social status (Yoo \& Lee 2018). Due to its advantages of easy communication, empathy, and security, peer education is a useful health education method that adolescents are willing to accept. This method has been recognized by the World Health Organization as an effective way to change people's behavior. In recent years, peer education has been widely used as a way of peer-to-peer health education in the prevention and treatment of AIDS, cancer, and chronic diseases, and it has achieved good results (Menna et al. 2015, Sharif et al. 2019).

The above literature shows that adolescents' anxiety and depression during the COVID-19 pandemic cannot be ignored. Adolescents are prone to negative psychological problems, such as anxiety and depression, during crisis events. Many researchers provided suggestions on psychological adjustment from different aspects, but only a few conducted targeted intervention studies. Therefore, we innovatively propose model 328 in this study and combine it with the psychological support form of peer education that is easily accepted by the adolescent group to intervene in the anxiety, depression, and sleep problems of young people during the COVID-19 pandemic. Through this study, we aim to provide reference on improving adolescents' mental health level during this crisis event.

\section{SUBJECTS AND METHODS}

\section{Participants}

In February 2020, we used a convenience sampling method via online platforms to investigate a total of 1,200 female and male middle school students (who are currently enrolled as Junior One, Junior Two, Senior One, and Senior Two students) aged 12 to 18 years in the provinces of Hunan and Guangxi in China. The participants in this experiment had already understood the purpose and method of the experiment before they proceeded, and all of them participated voluntarily in the study.

\section{Methodology}

Through the first round of an online electronic questionnaire survey, we randomly divided a total of 150 middle school students who volunteered to participate in the intervention experiment (with an anxiety score greater than 50 points) into control and intervention groups ( 75 members in each group). On the basis of the proposed routine treatment, we conducted 8 weeks of model 328-based peer education intervention 
in the intervention group. All the participants understood the purpose and basic operation methods of this study and volunteered to participate in the intervention. All the scales were filled in anonymously.

Peer education was adopted in the intervention group. We first conveyed health knowledge and behaviors concerning COVID-19 to those students with high enthusiasm for participating in the study through group discussion, role-playing, and games. Then, we asked them to educate their peers by communicating with them about COVID-19. We encouraged them to engage in peer education among classmates and friends by organizing weekly peer-education seminars in the form of online live webcasts. In accordance with model 328 , we selected aerobics in this study as an intervention program, which lasted for 8 weeks. The principle of model 328 is exercising 3 days a week scheduled every other day to ensure enough time for the body to recover. Exercising twice a day can guarantee the basic exercise time, and the arrangement of 8 movements (including 7 movements at major parts and 1 relaxing and finishing movement) each time can ensure the appropriate amount of exercise and exercise intensity. The designed exercise intervention program covered: 1. Exercise frequency: Exercises were scheduled 3 days per week, 2 times each day. 2. Exercise time: The duration of each exercise was 10 minutes. 3. Exercise intensity: Moderate intensity was set according to the adolescents' physique standards. Heart rate was controlled between $(220 \text {-age })^{*}(40$ $80 \%$ ). We sent a sports heart rate watch to each student in the intervention group through express delivery to measure their heart rate and ensure the accuracy of the study. Heart rate was mainly monitored by measuring the pulse immediately after an exercise to maintain moderate exercise intensity. 4. Exercise content: We formulated the content of the movements according to model 328. The movements were required to be simple and easy to master and meet the adolescents' physical characteristics. We recorded the movements and then delivered to the email addresses of the intervention group members in an MP4 format.

Routine health education was adopted in the control group. The intervention group and the control group received the same knowledge of health education, including diet and nutrition, lifestyle habits, physical exercise, mental health, and pandemic-related knowledge.

\section{Measuring Tools}

\section{Self-designed questionnaire}

The questionnaire includes two parts: the first part is about the participants' general demographic data, including their gender, age, professional background, grade, and physical condition; the second part is about their home living conditions, including 20 items measuring sleep time and regularity, dietary structure and regularity, activity intensity, pandemic concern, and exercise time.

\section{Self-rating anxiety scale (SAS) (Zung 1971)}

SAS was compiled by Zung (1971) and contains 20 test items and 4 grade scores. It mainly evaluates the participants' anxiety status measured with an interval of one week. SAS can well reflect the subjective feelings of the participants for one week. It is suitable for people of all occupations, cultures, and ages and adults with anxiety symptoms, and it has a wide range of applications.

\section{Self-rating depression scale (SDS) (Zung 1965)}

SDS was compiled by Zung (1965) and used to evaluate the participants' depression status and severity. This scale is filled in according to their feelings for one week. It can also intuitively reflect their subjective feelings. SDS includes 20 items in 4 dimensions, including psychotic emotional symptoms, physical disorder, psychomotor disorder, and psychological disorder of depression. This scale is simple and easy to master and suitable for people without mental health problems and mental patients of various occupations, cultures, and ages. The lower the total score obtained by the participants, the better their situation.

\section{Pittsburgh sleep quality index (PSQI) (Buysse et al. 1989)}

PSQI is a self-rating sleep quality scale compiled by Buysse et al. (1989) and one of the most widely used sleep quality assessment scales. The original form was translated into Chinese by Liu et al. (1996), tested for reliability and validity, and proven to be suitable for the use in China. It is applied to assess the participants' sleep quality for a month. It is suitable for people with sleep disorder and also for evaluating the sleep quality of those without sleep disorder.

\section{Statistical Analysis}

We analyzed the data by using SPSS 16.0 statistical software. We expressed the quantitative data as mean \pm standard deviation. We adopted independent sample $t$ test for cross-group comparison of the means of the two groups and used paired $t$ test for inter-group comparison. We expressed the qualitative data through the number of cases. Finally, we conducted chi-square test or rank sum test for cross-group comparison. $\mathrm{P}<0.05$ was considered to be statistically significant.

\section{RESULTS}

\section{General data}

Before the intervention, the intervention and the control groups had 75 members each. After the intervention, we lost contact with 5 members in the intervention group and 4 in the control group. We included a total of 70 members in the intervention group and 71 in the control group in the analysis. Table 1 shows that the two groups were not statistically and significantly different in terms of the participants' age, sex, grade, pandemic concern, exercise time, and sleep time $(\mathrm{P}>0.05)$, suggesting that they were comparable in the general data. 
Table 1. Comparison of the general data of the participants

\begin{tabular}{|c|c|c|c|c|}
\hline General information & Intervention group $(\mathrm{n}=70)$ & Control group $(\mathrm{n}=71)$ & $\mathrm{t} / \chi^{2} / Z$ & $\mathrm{P}$ \\
\hline Age & $15.2 \pm 2.1$ & $15.3 \pm 2.4$ & 0.263 & 0.793 \\
\hline Sex & & & 0.707 & 0.791 \\
\hline Male & 40 & 39 & & \\
\hline Female & 30 & 32 & & \\
\hline Grade & & & 0.759 & 0.859 \\
\hline Grade one of junior high school & 21 & 17 & & \\
\hline Grade two of junior high school & 19 & 22 & & \\
\hline Senior high school freshmen & 15 & 17 & & \\
\hline Senior high school sophomore & 15 & 15 & & \\
\hline Epidemic situation concern & & & 0.325 & 0.745 \\
\hline Very Concerned & 16 & 19 & & \\
\hline More Concerned & 29 & 21 & & \\
\hline Commonly & 16 & 21 & & \\
\hline Not Concerned & 9 & 10 & & \\
\hline Exercise time & & & 0.331 & 0.741 \\
\hline$>2 \mathrm{~h} / \mathrm{d}$ & 11 & 9 & & \\
\hline $1 \sim 2 \mathrm{~h} / \mathrm{d}$ & 12 & 13 & & \\
\hline $0.5 \sim 1 \mathrm{~h} / \mathrm{d}$ & 24 & 31 & & \\
\hline$<0.5 \mathrm{~h} / \mathrm{d}$ & 23 & 18 & & \\
\hline Sleep time & & & 1.356 & 0.175 \\
\hline$>9 \mathrm{~h} / \mathrm{d}$ & 21 & 27 & & \\
\hline $7 \sim 9 \mathrm{~h} / \mathrm{d}$ & 32 & 33 & & \\
\hline$<7 \mathrm{~h} / \mathrm{d}$ & 17 & 11 & & \\
\hline
\end{tabular}

Table 2. Comparison of SAS scores before and after the intervention

\begin{tabular}{lcccc}
\hline Group & Before intervention & After intervention & $\begin{array}{c}\text { Difference before and } \\
\text { after intervention }\end{array}$ & $\begin{array}{c}\text { Intra group } \\
\text { comparison p value }\end{array}$ \\
\hline Intervention group & $62.49 \pm 9.33$ & $55.00 \pm 10.49$ & $7.49 \pm 5.39$ & $<0.001$ \\
Control group & $63.39 \pm 9.77$ & $60.37 \pm 9.65$ & $3.03 \pm 3.68$ & $<0.001$ \\
$\mathrm{t}$ & 0.180 & 3.848 & 5.327 & \\
$\mathrm{P}$ & 0.857 & $<0.001$ & $<0.001$ & \\
\hline
\end{tabular}

Table 3. Comparison of SDS scores before and after the intervention

\begin{tabular}{lcccc}
\hline Group & Before intervention & After intervention & $\begin{array}{c}\text { Difference before and } \\
\text { after intervention }\end{array}$ & $\begin{array}{c}\text { Intra group } \\
\text { comparison p value }\end{array}$ \\
\hline Intervention group & $60.60 \pm 7.61$ & $53.83 \pm 9.52$ & $6.77 \pm 5.90$ & $<0.001$ \\
Control group & $62.30 \pm 8.61$ & $58.51 \pm 9.63$ & $3.79 \pm 4.50$ & $<0.001$ \\
$\mathrm{t}$ & 1.238 & 2.900 & 3.377 & \\
$\mathrm{P}$ & 0.218 & 0.004 & 0.001 & \\
\hline
\end{tabular}

\section{Comparison of SAS scores before and after the intervention}

Table 2 shows that the two groups before the intervention were not statistically and significantly different from each other in terms of SAS scores $(t=0.180$, $\mathrm{P}=0.857$ ). After 2 months of intervention, the SAS scores of the two groups both decreased, and the differences were statistically significant compared with those before the intervention $(\mathrm{P}<0.001)$. From the comparison of the SAS scores of the two groups after the intervention and the decreases in their SAS scores before and after the intervention, the effect on the intervention group was better than the control group, and the difference was statistically significant $(\mathrm{t}=3.948$ and 5.327, respectively; $\mathrm{P}<0.001$ ).

\section{Comparison of SDS scores before and after the intervention}

Table 3 shows that the two groups before the intervention were not statistically and significantly different from each other in terms of SDS scores $(\mathrm{t}=1.238$, $\mathrm{P}=0.218$ ). After 2 months of intervention, the SDS scores of the two groups both decreased, and the differences were statistically significant compared with those before the intervention $(\mathrm{P}<0.001)$. From the comparison of the SDS scores of the two groups after the intervention and the decreases in their SDS scores before and after the intervention, the effect on the intervention group was better than the control group, and the difference was statistically significant $(\mathrm{t}=2.900$ and 3.377 , respectively; $\mathrm{P}=0.004$ and 0.001 , respectively). 
Table 4. Comparison of the total PSQI scores before and after the intervention

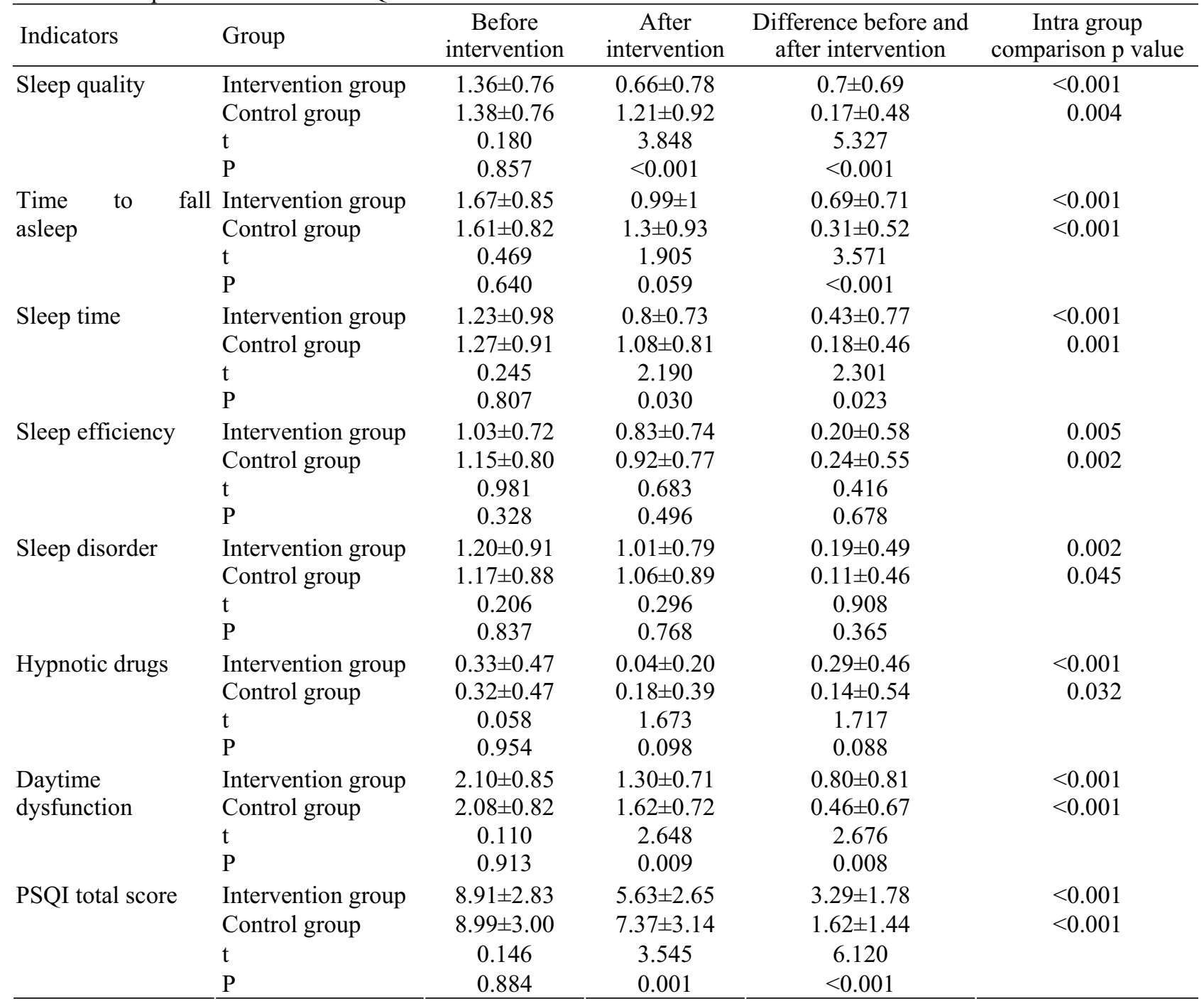

\section{Comparison of the total PSQI scores before and after the intervention}

Table 4 shows that the two groups before the intervention were not statistically and significantly different from each other in terms of the total PSQI scores $(t=0.146, P=0.884)$. After 2 months of intervention, the total PSQI scores of the two groups both decreased, and the differences were statistically significant compared with those before the intervention $(\mathrm{P}<0.05)$. From the comparison of the total PSQI scores of the two groups after the intervention and the decreases in their total PSQI scores before and after the intervention, the effect on the intervention group was better than the control group, and the difference was statistically significant $(\mathrm{t}=3.545$ and 6.120 , respectively; $\mathrm{P}=0.001$ and $<0.001$, respectively). From all dimensions, the four indicators, namely, sleep quality, time to fall asleep, sleep time, and daytime dysfunction, are consistent with the total PSQI scores. Moreover, the two groups were not different in terms of sleep efficiency, sleep disorder, and hypnotic drugs $(\mathrm{P}>0.05)$.

\section{DISCUSSION}

According to the results of comparison of general data, the 70 participants in the intervention group are not significantly different from the 71 participants in the control group in terms of their general data, including age, gender, grade, pandemic concern, exercise time, and sleep time. This result guarantees the consistency of the two sets of additional control variables in the intervention experiment design and the accurate interpretation of the research results. The participants included in this study are Junior One, Junior Two, Senior One, and Senior Two students, that is, nongraduating middle school students. The reason for choosing non-graduating students mainly lies in the following considerations: First, obvious differences exist between non-graduating and graduating students in academic tasks and perceived academic pressure. Studies show that academic pressure reported by graduating classes, especially Senior Three graduates, is significantly higher than other grades (Zhao \& Zhang 2008). Their emotional problems, such as depression and anxiety, are also more prominent and serious than 
other grades (Cui et al. 2018). Second, as graduate students are faced with the task of seeking higher education (in China, Junior Three and Senior Three students are in the stage of preparing for the senior high school entrance examination and the college entrance examination, respectively), they do not have high enthusiasm to participate in the intervention, which may result in a high rate of loss and insufficient samples that may affect the accuracy of the study results (Wang et al. 2013). Third, a mixed study of graduate and non-graduate students may affect the accuracy and validity of the study results, given that the influence of additional variables cannot be ruled out (Zhang et al. 2013). In addition, $60.28 \%$ of the participants are concerned and extremely concerned about the pandemic, indicating that most adolescents are deeply affected by the pandemic. This finding illustrates the necessity of paying attention to adolescents' mental health.

\section{Model 328-based peer education can reduce adolescents' anxiety during the COVID-19 pandemic}

According to the results of this study, the intervention and the control groups are not significantly different from each other in terms of anxiety score before the intervention. In accordance with the criterion of identifying anxiety when the score is greater than 50 points, all the participants suffer from mild anxiety. After the intervention combining physical exercise with peer education, the decrease of the intervention group in terms of anxiety levels is significantly higher than that of the control group. This result is consistent with previous studies on exercise and peer education interventions in other groups' anxiety levels (Jia et al. 2018). The main reason why model 823 can reduce anxiety is that after regular exercise, adolescents have formed a good habit of insisting on exercise to an extent, thereby improving their physical vision, that is, individual awareness of health-related knowledge (Li \& Liu 2018). This positive awareness is conducive to the improvement of adolescents' self-confidence in dealing with the COVID-19 pandemic, reducing their anxiety caused by this crisis. Peer education also reduces anxiety effectively. According to social support theory, peers are sources of support for individuals in terms of handling stressful life events. Equal and smooth exchange of knowledge and information between peers helps individuals develop comprehensive and positive awareness of the pandemic (Meehan et al. 2017). Peers can also vent emotions and comfort one another, thereby directly reducing their anxiety levels (Ho et al. 2017).

Although the anxiety level is significantly reduced after the intervention, the anxiety level of the middle school students is still greater than 50 points after a month of intervention. This finding indicates that the anxiety level of the middle school students is still high. On the one hand, the pandemic has not ended, and given the influence of overseas outbreaks, China is still faced with recurrence of the pandemic and even the risk of a comeback. Hence, the pressure caused by the COVID-19 pandemic still exists in a large social environment. No adolescent can escape from this environment. Although primary and secondary schools nationwide have opened one after another, the school as a place where people gather is still a high-risk area for outbreaks. Consequently, schools have adopted various prevention measures, such as widening the gap between desks and displaced dining. However, these measures also convey a negative psychological hint to middle school students (Zhu et al. 2020). On the other hand, as far as this study is concerned, the intervention time is only 2 months, and the absolute time is short. Capron \& Schmidt (2016) pointed out that emotional problems, such as anxiety and depression, are easy to repeat. Interventions within 3 months may eliminate symptoms in a relatively short period of time, but it is not enough for individuals to completely get rid of the negative emotional experience. Some researchers also revealed that peer education intervention should not be less than 6 months to make the participants thoroughly form healthy living habits.

\section{Model 328-based peer education can reduce adolescents' depression during the COVID-19 pandemic}

According to the results of this study, the intervention effect of model 328-based peer education on reducing adolescents' depression is consistent with the effect on reducing anxiety. First, the intervention and the control groups have high levels of depression before the intervention. The decrease in the level of depression in the intervention group after the intervention is significantly higher than that of the control group. This result is consistent with the finding obtained by Sun et al. (2016). Although anxiety and depression are different in terms of diagnostic criteria and clinical symptoms, they are still interrelated. Li (2013) studied the structure of depression and anxiety in Chinese adolescents, finding that the main factors influencing middle school students' anxiety include worry and allergies, fear, anxiety, and physiological symptoms, whereas the core components of depression include helplessness, sense of worthlessness, bad mood, and physiological symptoms. The emergence of COVID-19, especially with the absence of vaccine at this stage and the extremely strong interpersonal infectious characteristic, brings adolescents a deep sense of helplessness. Improving physical fitness through physical exercise increases adolescents' positive vision of their bodies, helps in forming positive self-awareness, and enhances their self-confidence. In this way, they can effectively alleviate the sense of helplessness and reduce their depression levels (Guo et al. 2017). Peer education can also maximize the subjective initiative of the intervention participants, encourage middle school students to participate in the intervention, and gradually cultivate a good learning and education atmosphere, thereby forming a complete 
and systematic health education model. It corrects middle school students' misunderstanding about the pandemic. Finally, social support from peer education reduces adolescents' sense of worthlessness. Previous studies showed that adolescents often make social comparisons with their peers to judge their self-worth (Yin et al. 2017). Peer education provides conditions for adolescents to make peer comparisons and reduces their sense of worthlessness.

According to the results of this study, the anxiety and depression levels of the control group also decrease significantly after 2 months. Although the decreases are lower than those of the intervention group, routine drug treatment is still proven to have certain intervention effect. Immediately after the sudden public health incident of COVID-19, China launched nationwide mental health consultation, paying special attention to the mental health problems of adolescents (Chang et al. 2020). Adolescents' psychological problems, such as anxiety and depression, need to be identified in time and intervened according to the actual situation. By doing so, the aggravating trend of unhealthy emotional disorder can be reduced, and adolescents' mental health can be improved.

\section{Model 328-based peer education can improve adolescents' sleep quality during the COVID-19 pandemic}

According to the results of this study, routine intervention and model 328-based peer education can significantly improve the overall sleep quality of adolescents and relieve their sleep disorder. The effect on improving sleep quality, time to fall asleep, and daytime dysfunction is particularly significant. This result is consistent with previous research results (Gong et al. 2019). As previously mentioned, anxiety and depression have a common physiological symptom, namely, sleep disorder (Shanahan et al. 2014). Doubt and negation against self-worth and fear of the outside world reduce adolescents' self-esteem and self-efficacy (Dale et al. 2019). According to the biological-socialpsychological model, poor psychological state is often manifested through poor physical state, and sleep disorder is one of the manifestations. According to the theory of ego-depletion, an individual's psychological resources are limited. When anxiety and depression consume excessive psychological resources of an individual, his/her resources for other psychological activities are reduced. In this case, individuals need to recover psychological resources through other activities, including sleep (Ren \& Zhang 2014). The recovery of psychological resources through sleep helps individuals cope with the anxiety and depression caused by stressful life events, thereby breaking the vicious circle between emotional disorder and sleep disorder. Physical exercise at appropriate intensity can effectively wake up the body's sensitivity, send a signal of "need to rest" to the body, and adjust the level of related hormones in the body, which are beneficial to the adjustment of the biological rhythm of adolescents and the development of good work and rest habits (Ren \& Zhang 2014). Furthermore, due to the positive feedback and mutual support from peer education, the anxiety and depression levels of adolescents are reduced. The interaction between the two aspects ultimately reduces adolescents' physiological symptoms and improves their sleep quality. Improved sleep quality assists adolescents to recover psychological resources, which in turn helps them cope with negative life events.

\section{CONCLUSION}

We used model 328-based peer education in this study to conduct an intervention with 141 adolescents who were randomly divided into intervention and control groups. After 2 months of intervention, we found that model 328-based peer education could significantly reduce the level of anxiety and depression in adolescents and improve their sleep quality, providing a reference model on how to improve their mental health during the COVID-19 pandemic. This finding was helpful for the formulation of mental health plans for adolescents who live and study at home during the pandemic. It also suggested the necessity to combine exercise and psychological intervention, offering a new perspective. However, some deficiencies in this study should be considered. First, this study targeted nongraduating students, but attention should also be paid to the mental health problems of graduating students. Future research could target adolescents in graduating classes. Second, we conducted the intervention in this study for only 2 months under the pandemic period. The end of the intervention could lead to a decline in the effect. Future research could extend the intervention time until the end of the pandemic. Model 328-based peer education was adopted in this study. Future research could include the comparison with other psychological intervention or apply various intervention methods to improve the intervention effect.

\section{Acknowledgements:}

This work was supported by National social sciences Fund of China (19BTY017).

\section{Conflict of interest: None to declare.}

\section{Contribution of individual authors:}

Xueshi Ding conceived and carried out experiments, analyzed data, prepared the manuscript, and analyzed the results.

Jiwei Yao supervised the study, analyzed the results, and discussed the results. All authors have read and agreed to the published version of the manuscript. 


\section{References}

1. Bowles TV: The focus of intervention for adolescent social anxiety: Communication skills or self-esteem. International Journal of School \& Educational Psychology 2017; 5:14-25

2. Brosnahan J, Steffen LM, Lytle L, Patterson J \& Boostrom A: The Relation Between Physical Activity and Mental Health Among Hispanic and Non-Hispanic White Adolescents. Archives of Pediatrics \& Adolescent Medicine 2004; 158:818-823

3. Buysse DJ, Reynolds CF, Monk TH, Berman SR \& Kupfer DJ: The Pittsburgh Sleep Quality Index: a new instrument for psychiatric practice and research. Psychiatry Research 1989; 282:193-213

4. Capron DW \& Schmidt NB: Development and randomized trial evaluation of a novel computer-delivered anxiety sensitivity intervention. Behaviour Research and Therapy 2016; 81:47-55

5. Chang JH, Yuan YX, Wang D: Mental health status and its influencing factors among college students during the epidemic of COVID-19. Journal of Southern Medical University 2020; 40:171-176

6. Danese A, Smith P, Chitsabesan P \& Dubicka B: Child and adolescent mental health amidst emergencies and disasters. The British Journal of Psychiatry 2020; 216:159-162

7. Davis JP, Dumas TM, Merrin GJ, Espelage DL, Tan K, Madden D, et al.: Examining the pathways between bully victimization, depression, academic achievement, and problematic drinking in adolescence. Psychology of addictive behaviors 2018; 32:605-616

8. Ghandour RM, Sherman LJ, Vladutiu CJ, Ali MM, Lynch SE, Bitsko RH, et al.: Prevalence and treatment of depression, anxiety, and conduct problems in US children. The Journal of pediatrics 2019; 206:256-267

9. Gong YJ, Zhang YF, Yang XY, Guan Y, Tang DH: Effectiveness of exercise intervention on sleep quality and negative emotion among female college students with anxiety. Chinese Journal of School Health 2019; 40:542545

10. Guan BQ, Luo XR, Deng YL, Wei Z, Ye HS, Yuan XH, et al.: Prevalence of psychiatric disorders in primary and middle school students in Hunan Province. Chinese Journal of Contemporary Pediatrics 2010; 12:123-127

11. Guo YF, Zhang X, Plummer V, Lam L, Cross $W$ \& Zhang JP: Positive psychotherapy for depression and selfefficacy in undergraduate nursing students: A randomized, controlled trial. International journal of mental health nursing 2017; 26:375-383

12. Holmes EA, O'Connor RC, Perry VH, Tracey I, Wessely $S$, Arseneault L, et al.: Multidisciplinary research priorities for the COVID-19 pandemic: a call for action for mental health science. The Lancet Psychiatry 2020; 7:547-560

13. Ho RT, Lai AH, Lo PH, Nan JK \& Pon AK: A StrengthBased Arts and Play Support Program for Young Survivors in Post-Quake China: Effects on Self-Efficacy, Peer Support, and Anxiety. The Journal of Early Adolescence 2017; 37:805-824

14. Jakovljevic M, Jakovljevic I, Bjedov $S$ \& Mustac F: psychiatry for better world: COVID-19 and blame games people play from public and global metal health perspective. Psychiatr Danub 2020; 32:221-228
15. Jia SN, Song YF, Meng C\& Zuo Q: Effect of early psychological intervention on maternal anxiety. Journal of China Medical University 2018; 47:458-460

16. Joseph SJ, Bhandari SS, Ranjitkar S, Duttal S: School closures and mental health concerns for children and adolescents during the covid-19 pandemic. Psychiatr Danub 2020; 32:309-310

17. Juan J, Gil MM, Rong Z, Zhang Y, Yang H, Poon LC: Effects of coronavirus disease 2019 (COVID) on maternal, perinatal and neonatal outcomes: a systematic review. Ultrasound in Obstetrics and Gynecology 2020; 56:15-27

18. Ramirez FE, Nedley N, Peterson D, Fukuda M, Emerson $J$ : Exercise Intervention with Lifestyle Improves Mental Health and Decreases Morbid Thoughts. Medicine \& Science in Sports \& Exercise 2018; 50:49-50

19. Cui J, Xin ZL, Ma L, Wang Z, Wang M, Wang YJ: Investigation of anxiety, depression and comorbidity among leftbehind primary school pupils and normal pupils in Harbin. Chinese Journal of Child Health Care 2018; 26:758-761

20. Li QQ, \& Liu Y: The causes of body image disturbance: An explanation from "looking-glass self" perspective. Advances in Psychological Science 2018; 26:117-127

21. Li YX: The Emotion of Depression and Anxiety of Adolescents and Their Relative Factors. China Journal of Health Psychology 2013; 21:415-417

22. Liu XC, Tan MQ, Hu L, Wang AZ, Wu HX, Zhao GF, et al.: eliability and validity of Pittsburgh sleep quality index. Chinese Journal of Psychiatry 1996; 29:103-107

23. Lubans DR, Aguiar EJ, Callister R: The effects of free weights and elastic tubing resistance training on physical self-perception in adolescents. Psychology of Sport \& Exercise 2010; 11:497-504

24. Mason BL, Davidov A, Minhajuddin A \& Trivedi MH: Focusing on insomnia symptoms to better understand depression: a star*d report. Journal of Affective Disorders 2019; 260:183-186

25. Meehan M, Massavelli B \& Pachana N: Using attachment theory and social support theory to examine and measure pets as sources of social support and attachment figures. Anthrozoös 2017; 30:273-289

26. Menna T, Ali A, Worku A: Effects of peer education intervention on HIVIAIDS related sexual behaviors of secondary school students in Addis Ababa, Ethiopia: a quasiexperimental study. Reproductive Health 2015; 12:1-8

27. Pekrun R: Emotion and achievement during adolescence. Child Development Perspectives 2017; 11:215-221

28. Ren $K \&$ Zhang LW: Application of the theory of ego depletion in the sports area. Journal of Physical Education 2014; 22:110-113

29. Shanahan L, Copeland WE, Angold A, Bondy CL \& Costello EJ: Sleep problems predict and are predicted by generalized anxiety/depression and oppositional defiant disorder. Journal of the American Academy of Child \& Adolescent Psychiatry 2014; 53:550-558

30. Sharif F, Abshorshori N, Tahmasebi S, Hazrati M, Zare N \& Masoumi S: The effect of peer-led education on the life quality of mastectomy patients referred to breast cancerclinics in Shiraz, Iran 2009. Health and Quality of Life Outcomes 2019; 8:1-7

31. Stavropoulos V, Gomez R, Steen E, Beard C, Liew L \& Griffiths MD: The longitudinal association between anxiety and Internet addiction in adolescence: The moderating effect of classroom extraversion. Journal of behavioral addictions 2017; 6:237-247 
32. Stein SF, Ngo QM, Austic EA, Veliz PT, McCabe SE \& Boyd CJ: The Clinical Relevance of Divergence in Adolescent-Parent Reports of Adolescent Depression and Anxiety. Child and adolescent social work journal 2018; 35:611-623

33. Sun X, Ren X, Cao $Y$ \& Wang Z: The effect of group psychological counseling and peer education on depressive and anxiety symptoms of pregnant females with selective multiple pregnancy reduction in second trimester. Chinese Journal of Practical Nursing 2016; 32:1215-1219

34. Tuliao KV, Chen C \& Yeh Y: Cross-national assessment of the effects of income level, socialization process, and social conditions on employees' ethics. Business Ethics: A European Review 2020; 29:333-347

35. Tull MT, Lee AA, Geers AL, Gratz KL: Exploring the role of sedentary behavior and physical activity in depression and anxiety symptom severity among patients with substance use disorders. Mental Health \& Physical Activity 2018; 14:98-102

36. Wang ZY, Wang JP, Andreas M: An internet-based selfhelp intervention program application for traumatized persons. Chinese Mental Health Journal 2013; 27:28-35

37. Xu R: On the promotion of "328 sports mode" from the perspective of national fitness. Sports World (Scholarly) 2019; 6:39-40

38. Yeh ZT, Huang YH \& Liu SI: Maternal depression and adolescent emotions: The role of family functioning. Journal of child and family studies 2016; 25:2189-2200
39. Yin XQ, Wang LH, Zhang GD, Liang XB, Li J, Zimmerman $M A$, et al.: The promotive effects of peer support and active coping on the relationship between bullying victimization and depression among Chinese boarding students. Psychiatry research 2017; 256:59-65

40. Yoo HH, Lee SY: Evaluation of effectiveness of peer education on smoking behavior among high school students. Saudi Medical Journal 2018; 39:635-637

41. Zhang Y, Song JP, Qi XC, Yang HJ, Zhao WQ \& Yang ZR: Surveys of mental health status in graduation and nongraduation class students of senioRmiddle school. Journal of Clinical Psychosomatic Diseases 2013; 19:144-145

42. Zhao YF, Shi L: Research on the Effect of Different Exercise Program Interventionon College Students with Depression. Journal of Guangzhou Sport University 2016; 36:93-97

43. Zhao ZQ \& Zhang H: On the sports and reduction of the mental stress of junior high school graduating graders. Journal of Hebei Normal University (Educational Science) 2008; 10:58-61

44. Zhu XL, Liu D, Yan F, Qu W, Fan HZ, \& Zhao YL, et al.: Psychological status of school students and employees during the COVID-19 epidemic. Chinese Mental Health Journal 2020; 34:549-554

45. Zung WW: A rating instrument for anxiety disorders. Psychosomatics 1971; 12:371-379

46. Zung $W W$ : A self-rating depression scale. Archives of General Psychiatry 1965; 12:63-70

Correspondence:

Jiwei Yao, MD

School of Sports and Health, Guangxi Normal University

Guilin, Guangxi, 541004, China

E-mail: yjwdxs09141020@163.com 